\title{
Electron Microscopic Observations of Chlamydia- like Microorganism in Hepatopancreas Cells of the Spider, Coras luctuosus
}

\author{
Haruki ÔSAKI \\ Kumamoto City Senior High School Tsuboi-4-chôme, \\ Kumamoto 860, Japan
}

\begin{abstract}
Chlamydia-like microorganism was observed not only in the cytoplasm but also within the intracytoplasmic inclusions of hepatopancreas cells of the spider, Coras luctuosus. The reticulate body, which bears a resemblance to that of members of the genus Chlamydia, is generally spherical and approximately 370 to $800 \mathrm{~m} \mu$ in diameter; its cytoplasm contains ribosome-like particles as well as fine DNA-like fibrils, and is bounded by a double-layered cell wall and a cytoplasmic membrane. The frequent occurrence of pairs or clusters of reticulate bodies and of profiles with symmetrical constriction, suggests that the reticulate bodies multiply by fission. Maturation process of the cell is accomplished by condensation of DNA-like fibrils. The elementary body is spherical, from 320 to $360 \mathrm{~m} \mu$ in diameter, with an eccentrically placed electron-dense nucleoid. In addition, four kinds of unusual parasites, which are undoubtedly cellular and procaryotic, are found only in the spider infected with the chlamydia-like microorganism. The possible relations of these parasites to the chlamydia-like microrganism are discussed.
\end{abstract}

\section{Introduction}

It is well known that the microorganism in the genus Chlamydia passes through a complex life cycle in which the infectious elementary body is taken into the host cell by phagocytosis and is converted into a large reticulate body which multiplies by fission, then undergoes maturation to form elementary body (Armstrong and ReEd, 1964; Cutlip, 1970 ; Higashi, 1964, 1965). Fortunately, during the course of the electron microscopic study of the hepatopancreas of the spider, Coras luctuosus, the microorganism which bears a morphological resemblance to the known members of the genus Chlamydia has been noticed. The presence of such microorganism has not previously been reported in spider cells. The microorganism, since precise identification is unknown, is referred to as chlamydia-like microorganism. Furthermore, 
four kinds of unusual procaryotic parasites are observed only in the spider infected with the chlamydia-like microorganism. This report describes the ultrastructural configurations of the chlamydia-like microorganism and the unusual parasites.

\section{Material and Methods}

Adult female spiders, Coras luctuosus, were collected in the suburbs of Kumamoto City, from early January to early April in 1972. Small pieces of hepatopancreas or glandular mid-gut were fixed in cold 2 per cent osmium tetroxide buffered at pH 7.2 with veronal acetate (PALADE, 1952) for 2 hours. After fixation, the tissues were rapidly dehydrated through a graded concentration of cold ethyl alcohol, and embedded in epoxy resin by the method described by LUFT (1961). Thin sections were cut on LKB microtome with glass knives. The sections were mounted on uncoated copper mesh grids, and then stained with uranyl acetate (WATSON, 1958) and lead citrate (REYNOLDS, 1963). Observations were made by the Hitachi HU-11A electron microscope.

\section{Results}

Hepatopancreas of seventeen individuals of the spider, Coras luctuosus, was examined by electron microscopy. As the result, only two individuals were infected with the chlamydia-like microorganism. Hepatopancreas of the spider is composed of numerous caeca separated from each other by the interstitial cells. The epithelium of the caeca consists of two distinct cell types: absorptive cells and secretory cells. Of all the hepatopancreas cells studied, the secretory cell exhibited the greatest retention of the microorganism.

There can be little doubt that the microorganisms in Figs. 1 and 2 (Arabic numbers 1 through 3) correspond to the reticulate bodies of the members of the genus Chlamydia, and I shall refer to them as such. The reticulate bodies are observed not only in the cytoplasm but also within the intracytoplasmic inclusion. They are essentially spheroidal in shape, measuring about 370 to $800 \mathrm{~m} \mu$ in diameter, and bounded by a double-layered cell wall and a cytoplasmic membrane; their cytoplasm contains ribosomelike particles and fine DNA-like fibrils (Figs. 3 and 5). 
The frequent occurrence of pairs or groups of reticulate bodies and of profiles with prominent constrictions, suggests that the reticulate body multiplies by fission. Intercellular bridges exist as open communications between daughter cells and frequently remain until the cells are nearly mature (Fig. 7). The number of daughter cells which may be connected in this fashion cannot be ascertained from the study of thin sections. The largest number of daughter cells found associated in this manner has been three, but greater numbers are likely to be involved. In a single field I have frequently found two daughter cells interconnected by one intercellular bridge, each of which showed symmetrical constriction in the equatorial region (Fig. 4). The image in this figure suggests that deepening of these indentations toward each other could result in the formation of four attached daughter cells. In Fig. 5 one cell is shown connected by two bridges to two other cells, one of which shows shallow equatorial indentation. Clusters of three daughter cells connected by a three-forked bridge were occasionally observed (Fig. 6).

Maturation process of the reticulate body into the elementary body appears to be accomplished by condensation of the DNA-like fibrils. The fibrils become zonally condensed in several electron-dense patches (Figs. 4 and 6). The patches of nucleoid material progressively coalesce to form a large nucleoid (Fig. 7). In the conjoined daughter cells the condensation of nucleoid material appears to be synchronized and it is assumed that the protoplasmic continuity is the basis for this synchronism. The elementary body is principally spherical, from 320 to $360 \mathrm{~m} \mu$ in diameter, with an eccentrically placed nucleoid (Arabic number 4 in Figs. 1 and 2, and Fig. 5).

The most interesting feature of the hepatopancreas cells infected with the chlamydia-like microorganism is the presence of four kinds of unusual parasites. They are found not only in the intracytoplasmic inclusions but also within the cytoplasm.

(a) First kind is irregularly spherical, about 320 to $360 \mathrm{~m} \mu$ in diameter and hereafter designated as "S-parasites" (Figs. 1, 2 and 8). The S-parasite, made up of ribosome-like particles and a nucleoid, is structurally similar to the elementary body of the chlamydia-like microorganism. The electrondense nucleoid, which seems to be consisted of fine DNA-like fibrils, is extremely eccentric; it is always placed under a cone formed by the extrusion of the cell wall. They may be found singly or in clusters of two (SP in Fig. 1) to three (Fig. 8, inset) cells interconnected by the intercellular bridge. 
(b) Second kind is roughly oval, about $400 \times 250 \mathrm{~m} \mu$, and hereafter called "O-parasites" (Figs. 1 and 8). Their cytoplasmic membranes appear to be internally lined with a thick layer of nucleoid material, which incompletely surrounds the cytoplasm containing ribosome-like particles.

(c) Third is somewhat flattened, rod-shaped parasites, measuring about $500 \times 200 \times 150 \mathrm{~m} \mu$, and tentatively named “R-parasites" (Figs. 1, 2, 8 and 9). Their cytoplasm, which is almost completely enveloped by the fine fibrillar nucleoid material, contains ribosome-like particles which are identical in appearance to that present in the cytoplasm of the S- and O-parasites. Occasional intercellular bridges with several constrictions appear to contain agranular material of the same electron opacity as that present in the lumen of free, rounded vesicles (Fig. 9, inset). The detachment of these beads would result in the production of free vesicles.

(d) Fourth is remarkably flattened, rod-shaped parasites, measuring about $500 \times 210 \times 80 \mathrm{~m} \mu$, and referred to as "F-parasites" (Figs. 1, 2, 10-12). Moderately electron-opaque cytoplasm and a thin plate-like structure are almost entirely enveloped by the nucleoid material. The plate, about $4 \mathrm{~m} \mu$ in thickness, has the same electron density as the cytoplasmic ground substance, and it is surrounded by a zone or halo of electron transparency (Fig. 12). The cell wall of the organism has a total thickness of about $11 \mathrm{~m} \mu$ and consists of two dense layers, separated by a clear region. In favorable section the layers are regularly perforated (Fig. 12, inset). Generally, intracytoplasmic inclusion filled with many $\mathrm{F}$-parasites is completely surrounded by a myelin-like sheath (Figs. 1 and 12). The origin of the sheath is unknown. Figs. 10 and 11 suggest, however, that the sheath may be derived from cisternae or vesicles of the endoplasmic reticulum which are located around the inclusion. Such cisternae or vesicles of the endoplasmic reticulum probably fuse one another to form the sheath.

\section{Discussion}

Although mechanism by which chlamydia-like microorganism enters the hepatopancreas cells of the spider, Coras luctuosus, and immediate consequences thereof, have not been established, the intracellular mode of multiplication appears to be essentially similar to that reported for other chlamydiae. The general mode of replication for chlamydia-like microorganism is 
binary fission of the reticulate body. Maturation process of the cell is accomplished by condensation of fine DNA-like fibrils. The trefoil-shaped cluster of reticulate bodies appears to be interesting (Fig. 6), since no similar profile has not been reported heretofore in the known members of the genus Chlamydia. Reproductive mode of a large reticulate body to a trefoil-shaped cluster is not known. The cluster could not be explained by the binary fission.

The known members of the genus Chlamydia are the parasites of tissue cells of birds and mammals. The chlamydia-like microorganism, on the contrary, was found only in the spider infected with the unusual parasites, and its reticulate body appeared to be able to grow and multiply in the spider hepatopancreas. PAGE (1966) has described about natural habitat of the organisms of the genus Chlamydia as follows: "Natural interspecies transfers occur, but the range and extent of transfers are not known. Arthropods may be involved in the mechanical transmission of certain agents between hosts, but are not known to support the multiplication of any of the agents". Whether or not this chlamydia-like microorganism may be found in various species of spiders is not known, since, to our knowledge, this is the first report of electron-microscopic work on spider microorganisms. This finding, so diametrically opposed to general definition of the genus Chlamydia, suggests that another genus exists. Further studies with true spiders and their close relatives, not only of the hepatopancreas but also of the other digestive organ and of the Malpighian vessel should be rewarding.

Unusual parasites, so-called "S-, O-, R- and F-parasites", are undoubtedly cellular and procaryotic microorganisms which, to our knowledge, have never been previously described in arthropods. It must be noted that these parasites are never found in healthy spiders or hepatopancreas uninfected with the chlamydia-like microorganism, and that the intracytoplasmic inclusions containing chlamydia-like microorganism may contain all kinds of the parasites (Figs. 2 and 8). In general, a decrease in the amount of $\mathrm{S}$ - and/or Oparasites within an intracytoplasmic inclusion is concomitant with an increase in the amount of R-and/or F-parasites. Neither sign of cross-wall formation nor profile suggesting constriction was observed in all kinds of the parasites, except for occasional intercellular bridge.

From the above observations it is assumed that these parasites may constitute a single species of microorganism characteristic of the spider, although 
they lack an uniform structure. The different forms and sizes may be due to their morphological conversion which occurs in maturation process. If this conception is correct, there seems to be no serious objection to the idea that the $\mathrm{O}$ - and $\mathrm{R}$-parasites are intermediate form transforming into the $\mathrm{F}$ parasites or mature form from the S-parasites or juvenile form. As the elongation of the S-parasites proceeds, the nucleoid material, which is located under a cone formed by extrusion of the cell wall, moves down from this region along the cytoplasmic membrane until it reaches the another pole; subsequently, the cytoplasmic membrane of the R-parasites is internally lined with nucleoid material, which surrounds the cytoplasm. During late maturation, the parasites assume a flattened rod-shape, and a plate-like structure occurs in the cytoplasm.

Of particular interest are the S-parasites. If the extreme eccentricity of the nucleoid is disregarded, structural details of the S-parasites bear a surprising resemblance to those of the elementary body of the chlamydia-like microorganism. Furthermore, it may be significant that the clusters of two to three cells interconnected by an intercellular bridge are occasionally encountered in both microorganisms. These suggest that the S-parasites are derived from the elementary body of the chlamydia-like microorganism. If this is correct, then, it may be also conjectured that truly mature form of chlamydia-like microorganism is F-parasites. The studies on the nature of the S-parasites may be one of the most important subject in the future investigation.

The author wishes to express his hearty thanks to Dr. C. Ôra, Department of Anatomy, School of Medicine, Kumamoto University, for the generous use of his laboratory during the course of this study and invaluable help. He also would like to express his gratitude to Mr. T. Kubota for his help in taking the electron micrographs.

\section{References}

Armstrong, J. A., and S.E. Reed, 1964. Nature and origin of initial bodies in lymphogranuloma venereum. Nature, 201:371-373.

Cutlip, R. C., 1970. Electron microscopy of cell cultures infected with a chlamydial agent causing polyarthritis of lambs. Infec. Immun., 1: 499-502.

HigAshi, N., 1964. The mode of reproduction of the psittacosis-lymphogranulomatrachoma (PLT) group virus. Int. Rev. Expt1. Pathol., 3 : 35-64. 
Higashi, N., 1965. Electron microscopic studies on the mode of reproduction of trachoma virus and psittacosis virus in cell cultures. Expt1. Mol. Pathol., 4: 24-39.

LufT, J. H., 1961. Improvements in epoxy resin embedding methods. J. Biophys. Biochem. Cytol., 9: 409-414.

PAGE, L. A., 1966. Revision of the family Chlamydiaceae Rake (Rickettsiales) : unification of the psittacosis-lymphogranuloma venereum-trachoma group of organisms in the genus Chlamydia Jones Rake, and Stearns, 1945. Int. J. Syst. Bacteriol., $16: 223-252$.

Palade, G. E., 1952. A study of fixation for electron microscopy. J. Expt1. Med., $95: 285-298$.

REynolds, E.S., 1963. The use of lead citrate at high $\mathrm{pH}$ as an electron-opaque stain in electron microscopy. J. Cell Biol., $17: 208-212$.

Watoson, M.L., 1958. Staining of tissue sections for electron microscopy with heavy metals. J. Biophys. Biochem. Cytol., $4: 475-478$.

\section{Explanation of Figures}

Fig. 1. Relatively low magnification of an electron micrograph showing various developmental forms of the chlamydia-like microorganism in the secretory cell cytoplasm of hepatopancreas of the spider, Coras luctuosus. Process of the sequential development is illustrated by Arabic numbers 1 through 4. Arrows indicate intercellular bridges. FP, F-parasites; L, lipid droplets; OP, O-parasites; RP, R-parasites; S, secretion; SH, myelin-like sheath; SP, S-parasites. $\times 11,600$.

Fig. 2. A large intracytoplasmic inclusion in an absorptive cell. The inclusion cavity contains reticulate bodies ( 2 and 3 ) and elementary bodies (4) of the chlamydialike microorganism and three kinds of unusual parasites. FP, F-parasites; RP, R-parasites; SH, myelin-like sheath; SP, S-parasites. $\times 14,800$.

Fig. 3. Profiles of reticulate bodies within an inclusion cavity (IC), suggesting multiplication by fission. Note the intercellular bridge (arrow). IM, inclusion membrane. $\times 50,000$.

Fig. 4. Two daughter cells interconected by one intercellular bridge (long arrow). Note the shallow equatorial indentations (short arrows) and patchy aggregates of nucleoid material $(\mathrm{N}) . \quad \times 46,000$.

Fig. 5. Three daughter cells connected by two intercellular bridges (arrows). In the upper left-hand corner there is an elementary body which contains an electron-dense, eccentric nucleoid $(\mathrm{N})$. Two reticulate bodies with prominent constrictions are observed in the lower left part. $\times 53,000$.

Fig. 6. A cluster of three daughter cells connected by a three-forked bridge (arrow). $\mathrm{N}$, patches of nucleoid material. $\times 50,000$.

Fig. 7. The patches of nucleoid material coalesce to form an eccentric nucleoid $(\mathrm{N})$. Note the inclusion membranes (arrows). $\times 50,000$.

Fig. 8. A portion of intracytoplasmic inclusion containing reticulate bodies (RB) of the chlamydia-like microorganism, S-parasites (SP), O-parasites (OP), R-parasites (RP) and small vesicles (V). A small vesicular protrusion (long arrow), which represents remnant of intercellular bridge, appears to be pinching off 
from the surface of a S-parasite. Short arrows point to inclusion membrane. $\times 54,000$. Inset: A trefoil-shaped cluster of S-parasites. Note the nucleoid located under a cone-shaped extrusion. $\times 50,000$.

Fig. 9. Sections at various regions of the R-parasites are seen. Note the filamentous nucleoid material $(\mathrm{N})$, cytoplasm containing ribosome-like particles (R) and detached vesicles (arrows). $\times 50,000$. Inset: Beaded intercellular bridge. $\times 70,000$.

Fig. 10. A portion of intracytoplasmic inclusion in a secretory cell. Note narrow cisternae of the granular endoplasmic reticulum (arrows) just outside the inclusion membrane (IM). N, host cell nucleus; $\mathrm{SH}$, myelin-like sheath. $\times 44,000$.

Fig. 11. Beginning of formation of the myelin-like sheath in an absorptive cell. Small vesicles of the agranular endoplasmic reticulum (arrows) are located around the intracytoplasmic inclusions. $\times 25,000$.

Fig. 12. Longitudinal (L), cross (C) and nearly face (F) aspects of F-parasites, which are composed of engulfed cytoplasm (CY) of intermediate density, thin plate-like structure surrounded by the halo of electron transparency $(\mathrm{PL})$, thick layer of highly electron-dense nucleoid material (N) and double-layered cell wall $(\mathrm{CW})$. The cytoplasm appears to be continuous with the cytoplasmic membrane at the region indicated by the arrows. SH, myelin-like sheath. $\times 80,000$. Inset: High magnification view of nearly cross sections of F-parasites. The cell wall consists of two dense layers separated by a less dense region. The layers appear perforated in the regions indicated by the arrows. Cytoplasmic membranes are not distinguishable. $\times 200,000$. 


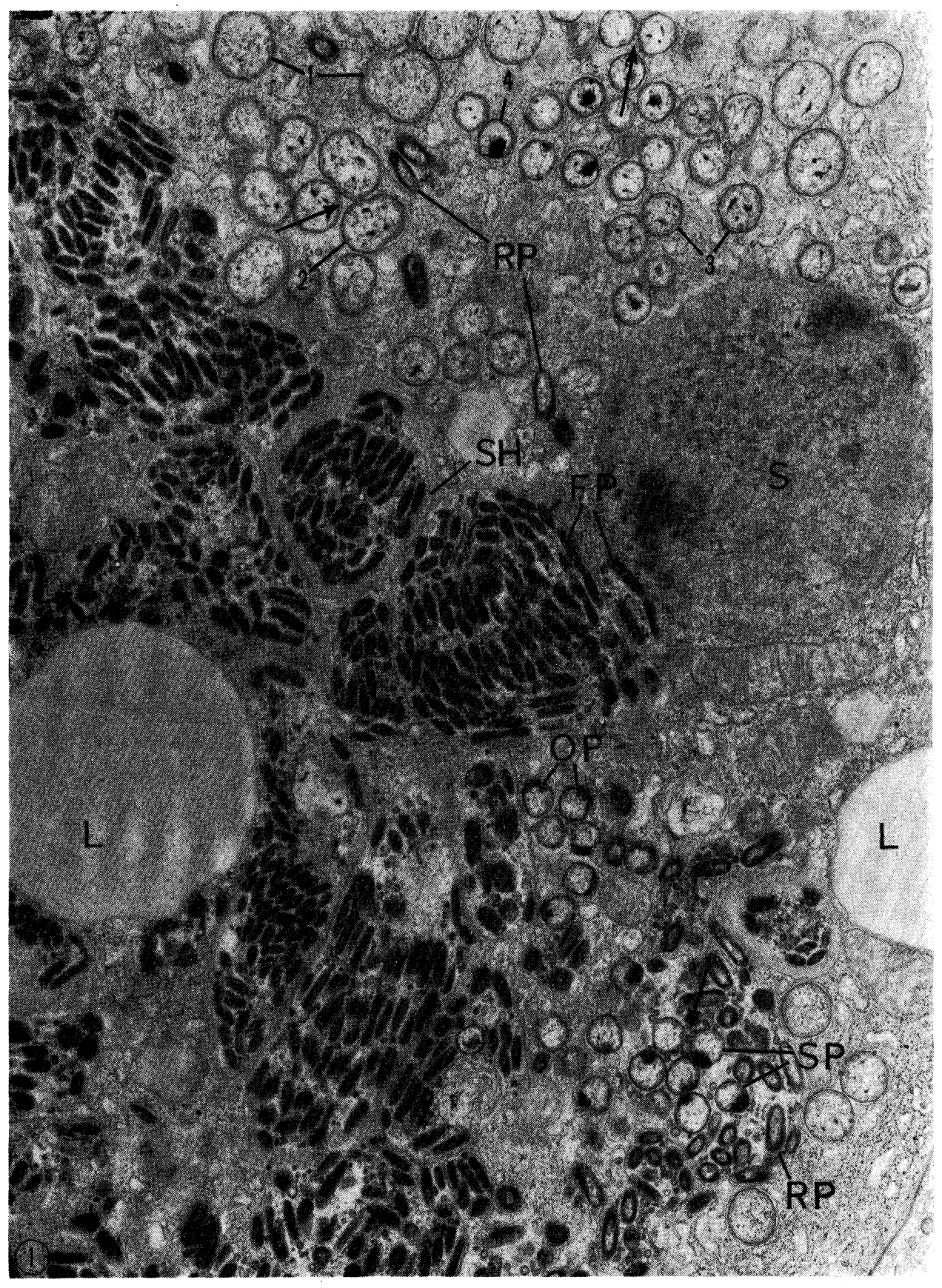




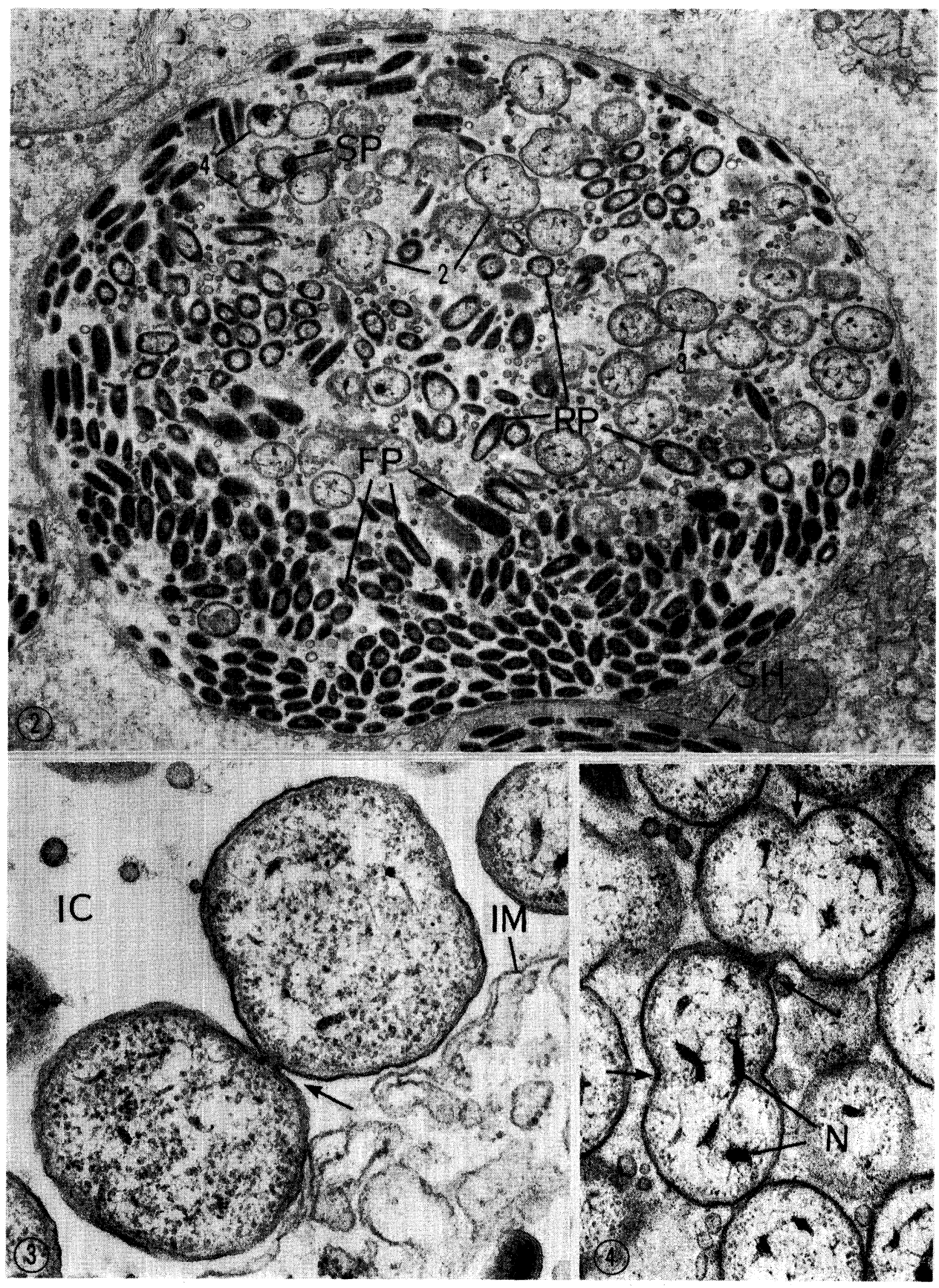




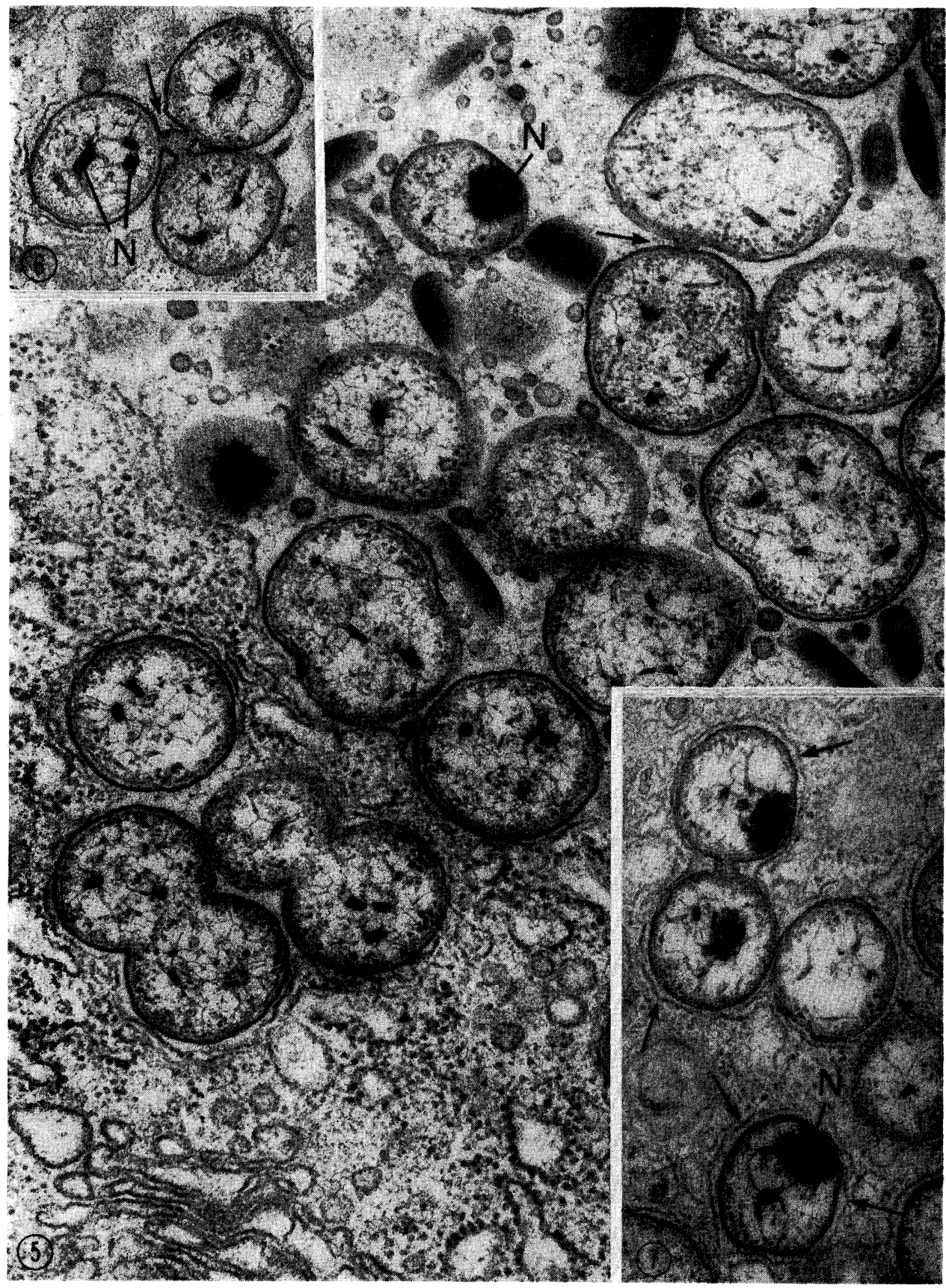




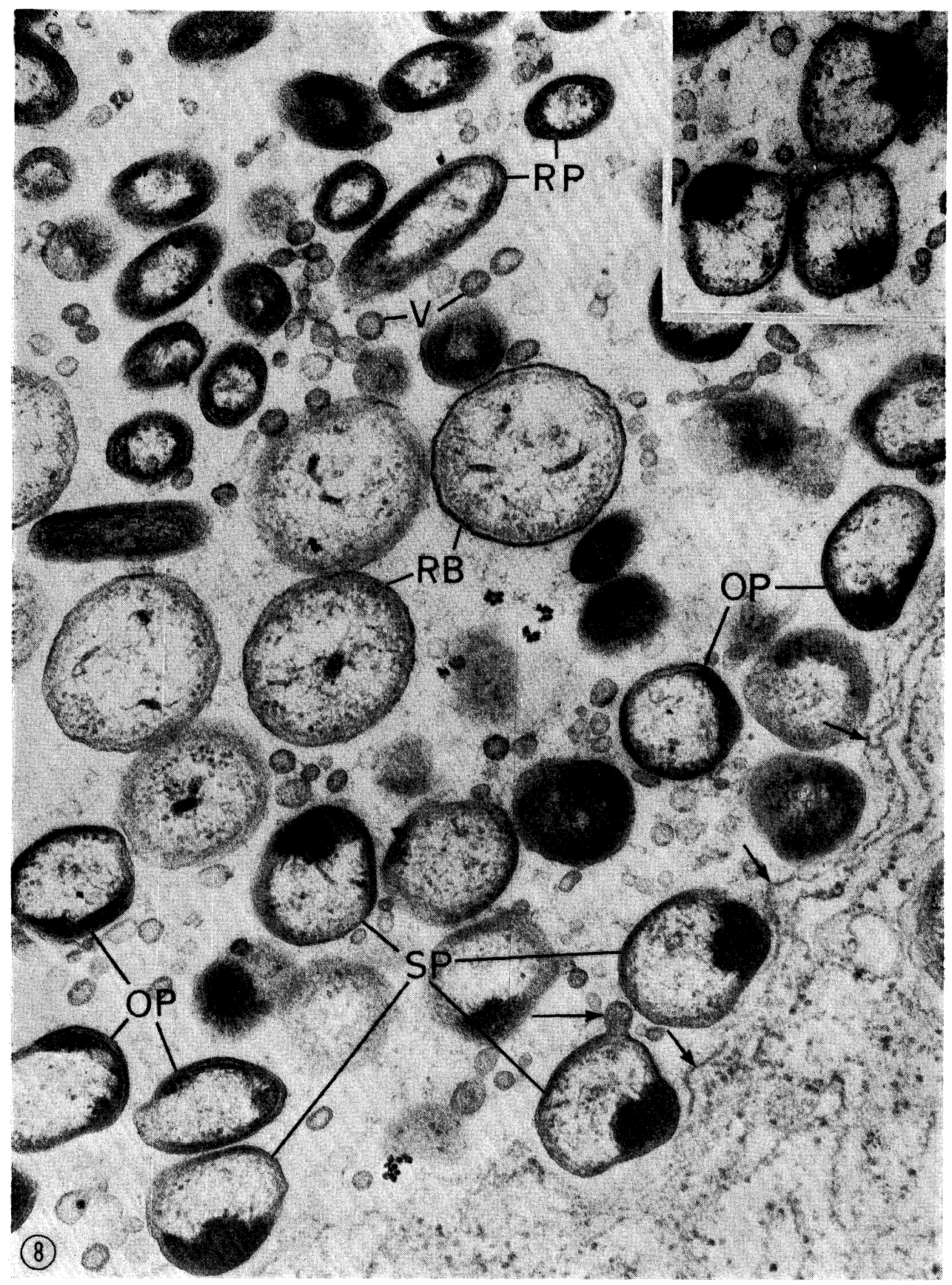




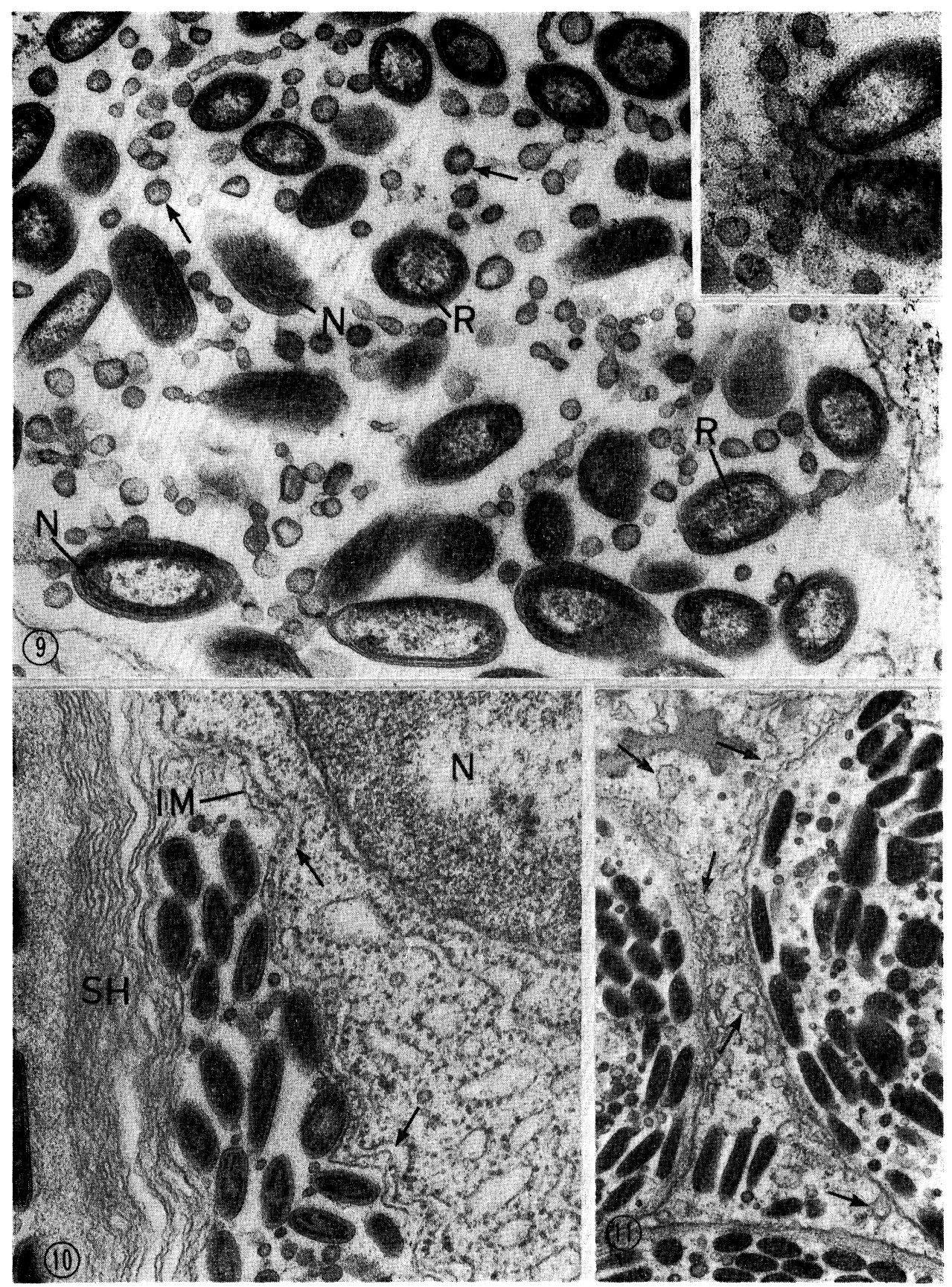



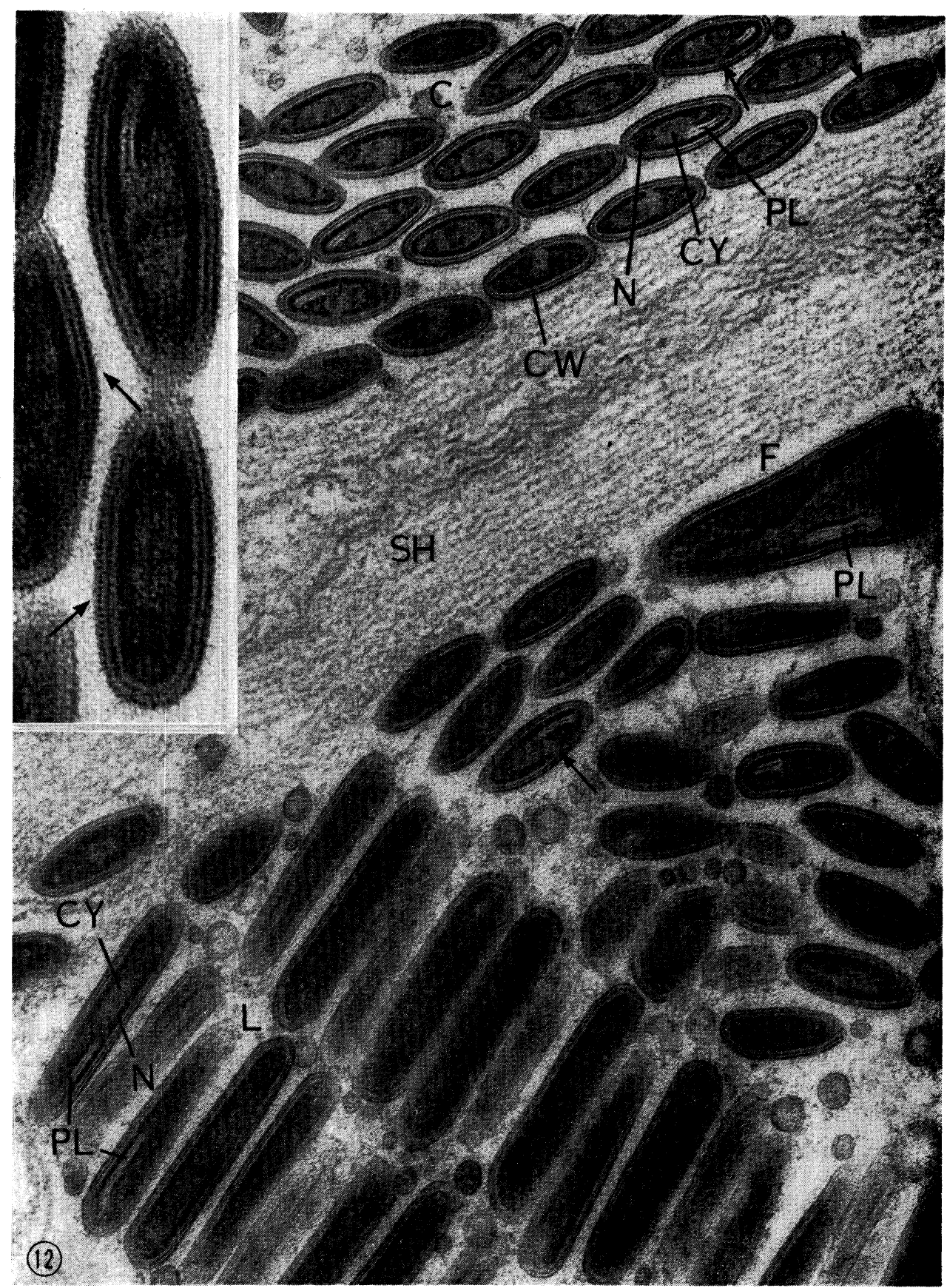

(12) 